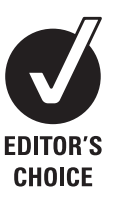
Medical University of South Carolina, Charleston, South Carolina, USA

2Department of Medicine, Medical University of South Carolina, Charleston, South Carolina, USA

${ }^{3}$ Department of Neurosciences, Medical University of South Carolina, Charleston, South

Carolina, USA

\section{Correspondence to} Dr Aquilla Turk, Medical University of South Carolina, 169 Ashley Ave, Charleston, South Carolina 29425, USA; m.cook@nhs.net

Received 27 April 2011 Revised 13 June 2011 Accepted 14 July 2011 Published Online First 19 September 2011

\title{
CT perfusion-guided patient selection for endovascular treatment of acute ischemic stroke is safe and effective
}

\author{
Aquilla Turk, ${ }^{1}$ Jordan Asher Magarik, ${ }^{2}$ Imran Chaudry, ${ }^{1}$ Raymond D Turner, ${ }^{3}$ \\ Joyce Nicholas, ${ }^{3}$ Christine A Holmstedt, ${ }^{3}$ Julio Chalela, ${ }^{3}$ Angela Hays, ${ }^{3}$ \\ Christos Lazaridis, ${ }^{3}$ Edward Jauch, ${ }^{3}$ Marc Chimowitz, ${ }^{3}$ Tanya Turan, ${ }^{3}$ Robert Adams ${ }^{3}$
}

\begin{abstract}
Background Traditional treatment in acute ischemic stroke is based on time criteria when administering intravenous and intra-arterial therapies. However, recent evidence suggests that image-based criteria may be useful for selecting patients for intra-arterial interventions. The use of CT perfusion (CTP)-based criteria, regardless of time from symptom onset, in patient selection for intra-arterial treatment of ischemic stroke was assessed.
\end{abstract}

Methods Patients with ischemic stroke who presented to the emergency department at the Medical University of South Carolina with a National Institute of Health Stroke Scale score of $\geq 8$, regardless of time from symptom onset, were assessed retrospectively. CTP maps were qualitatively assessed for the presence of penumbra and infarction. Selected patients underwent mechanical aspiration of their occlusion using the Penumbra system. Functional outcome was then recorded using the modified Rankin scale (mRS) at 90 days or the closest follow-up to 90 days.

Results 53 patients were included in the study. The median time from symptom onset to groin vascular access was $6.3 \mathrm{~h}$. Eight patients (15\%) had bleeding complications including subarachnoid hemorrhage, parenchymal hemorrhage and intraventricular hemorrhage. After CTP-based selection, the patients were divided into two groups for analysis: $\leq 6 \mathrm{~h}$ and $>6 \mathrm{~h}$ from symptom onset to endovascular procedure. No difference was found in functional outcome between the two groups $(38.5 \%$ and $40.7 \%$ achieved 90 -day mRS $\leq 2$, respectively $(p=1.0)$ and $57.7 \%$ and $51.9 \%$ achieved 90-day $\mathrm{mRS} \leq 3$, respectively $(p=0.785)$ ). There was no difference in the rate of intracranial hemorrhage between the two groups (11.5 vs $18.5, p=0.704$ ).

Conclusion This study demonstrated similar rates of good functional outcome and intracranial hemorrhage in patients with ischemic stroke when endovascular treatment was performed based on CTP selection rather than time-guided selection. These findings suggest that endovascular reperfusion in ischemic stroke may be effective and safe, and may allow patient selection not solely based on time from symptom onset.

\section{BACKGROUND}

Current recommendations for the recanalization of patients with acute ischemic stroke are based on time-defined parameters. Intravenous tissue plas- minogen activator ( $\mathrm{tPA}$ ) administration is indicated within 3 h of symptom onset, with newer evidence suggesting that tPA may be beneficial up to $4.5 \mathrm{~h}^{1-4}$ In addition, intra-arterial thrombolysis within $6 \mathrm{~h}$ has been shown to be effective in vessel recanalization and in improving patient outcomes while thrombectomy devices may be effective up to $8 \mathrm{~h}$ from symptom onset. ${ }^{5-10}$ Several recent reports have suggested that, with imaging-based selection, patients may be effectively treated beyond these strict time boundaries with good outcomes. ${ }^{11-14}$

To date, non-contrast head computed tomography (NCCT) is the principal imaging modality for suspected acute ischemic stroke. More recently the acquisition of qualitative physiologic data with CT perfusion imaging (CTP) has improved the sensitivity and specificity of identifying acute infarctions and penumbral regions. ${ }^{15-19}$ Current trials such as the mechanical retrieval and recanalization of stroke clots using embolectomy (MR RESCUE) and the efficacy and safety study of desmoteplace to treat acute ischemic stroke (DIAS3) are evaluating the utility of perfusion imaging to select patients with acute stroke for treatment without traditional time limitations. We report the experience at our center using CTP-based imaging to select patients with acute ischemic stroke for endovascular recanalization, irrespective of time constraints.

\section{MATERIALS AND METHODS}

An Institutional Review Board-approved retrospective review of a prospectively managed clinical database was performed for all cases of acute ischemic stroke intervention performed from 1 January 2008 to 31 December 2009. Chart review was further performed to assess the clinical course and outcome.

A retrospective chart review was performed through an electronic medical record. Patient characteristics documented included age, race, gender, National Institute of Health Stroke Scale Score (NIHSSS) at presentation, time to presentation from last normal and modified Rankin Score (mRS) at 90 days or closest follow-up period to 90 days. Modified Rankin Score data were obtained from the neurology clinic record. If the medical record did not contain outcome information, an independent neurologist contacted the patient or family to 
determine the functional status of the patient in order to determine the $\mathrm{mRS}$. Radiological and angiographic imaging were reviewed to document location of vascular occlusion, recanalization time, TIMI flow post-procedure, procedural complications and the presence of intracerebral hemorrhage post- or periprocedurally.

\section{Patient selection}

All stroke patients presenting with NIHSS $\geq 8$, irrespective of time of symptom onset, were considered candidates for endovascular therapy. All patients underwent NCCT, CT angiography (CTA) and CTP on admission to the emergency department. The NCCT scan was used to determine the presence and extent of gross infarction and to exclude underlying pathology such as mass or intracranial hemorrhage (ICH). CTA was used to identify the location of vascular occlusion. CTP was analyzed to identify the presence and extent of penumbra relative to the core area of infarction. The primary endpoints of the study were the percentage achieving good functional outcome as measured by 90 -day $\mathrm{mRS} \leq 2$ and $\mathrm{mRS} \leq 3$. The primary safety endpoints were symptomatic ICH (sICH) within $36 \mathrm{~h}$ and mortality at 90 days.

\section{CTP imaging techniques}

All CT imaging was performed according to a standardized institutional protocol. All CTP scans were performed using a Siemens 64- or 16-slice scanner with a $50 \mathrm{ml}$ Omnipaque 350 (GE Healthcare, Milwaukee, WI, USA) contrast bolus. Perfusion datasets were post processed on a GE Advantage Windows Workstation (General Electric; Milwaukee, Wisconsin, USA), Siemens Leonardo Workstation (Siemens Medical, Germany), or both. CTA was subsequently performed with an $80 \mathrm{ml}$ bolus injection of contrast followed by a saline chase. Two-dimensional $1 \mathrm{~cm}$ thick slab reformations were created in the axial, sagittal and coronal projections. These data were used to localize the location of the major vessel occlusion.

\section{Data processing}

CTP after processing on the GE workstation was performed by a radiology resident or by an experienced CT technologist yielding mean transit time (MTT), cerebral blood volume (CBV) and cerebral blood flow (CBF) maps. CTP after processing on the Siemens workstation was performed by an experienced CT technologist. MTT, CBV and CBF maps were reconstructed. The arterial input and venous outflow curves were analyzed to ensure complete data sets. All CTP interpretations for treatment were made by the attending neurointerventionalist based on qualitative interpretation of the perfusion maps. MTT maps were analyzed to define the area of brain at risk as delineated by at least two color band differences on the six-spectrum rainbow scale from the surrounding unaffected brain. CBV was evaluated to delineate the core region of infarction as the area with depressed CBV at least two color band differences on the sixspectrum rainbow scale from the surrounding unaffected brain. $\mathrm{CBF}$ was used to confirm these findings and further define the area at risk. Patients in whom one-third or more of the MCA territory was infarcted or with $\leq 50 \%$ penumbra were not considered candidates for endovascular treatment unless an eloquent area was at risk.

\section{Endovascular treatment}

Written informed consent for endovascular therapy was obtained in all cases. All cases were performed under general anesthesia. Patients received heparin in a 1500-unit bolus followed by a 500 -unit bolus every hour thereafter. The primary method of treatment in most cases was mechanical aspiration with the Penumbra aspiration system. The approach that evolved over the first 10 cases was to use the largest caliber aspiration catheter that the occluded vessel would accommodate. Intra-arterial thrombolysis with tPA was used adjunctively at low doses ranging from 10 to $20 \mathrm{mg}$ (administered in 3-5 mg aliquots) during the procedure if aspiration was not immediately effective. In patients who presented within the $0-3$ or $3-4.5 \mathrm{~h}$ time windows, intravenous tPA was administered to eligible patients before going to the angiography suite. Intra-arterial abciximab was administered if acute intraprocedural thrombus formation was felt to be present. If a permanent stent was used either to recanalize the thrombosed vessel or to treat a proximal stenosis, the patient was given a weight-based loading dose of abciximab and $325 \mathrm{mg}$ aspirin and $600 \mathrm{mg}$ clopidogrel were administered immediately after the procedure. All patients were managed postoperatively in the neurosurgical intensive care unit for at least the first $24 \mathrm{~h}$ after the endovascular procedure to ensure strict blood pressure monitoring and control.

\section{Data analysis}

Baseline demographic, radiographic and outcomes data were analyzed using descriptive statistics including means, standard deviations, frequencies, percentages and medians. Comparisons between time to treatment groups were made using two-sided $t$ tests for continuous measures, $\chi^{2}$ tests for categorical measures and Mann-Whitney $U$ tests for non-parametric measures (medians). No adjustments were made for multiple comparisons and $p$ values $<0.05$ were considered significant.

\section{RESULTS}

Fifty-three patients underwent endovascular treatment, with 18 receiving intravenous tPA prior to intra-arterial therapy. The overall mean age was 65 years (median 66 years) and $49 \%$ of patients were men. The mean NIHSS at presentation to our institution was 17.2 (median 17) and the locations of vascular occlusion were $22.6 \%$ internal carotid artery, $60.4 \%$ middle cerebral artery (MCA) and $17.0 \%$ posterior circulation (basilar artery or posterior inferior cerebellar artery) (table 1).

The mean time from the last point the patient was seen normal to groin vascular access was $10.4 \mathrm{~h}$ (median $6.3 \mathrm{~h}$, range 1.75-72 h). The mean time to obtain working access with a guide catheter from puncture was $20 \mathrm{~min}$ and to obtain TIMI II revascularization was $41.4 \mathrm{~min}$. Revascularization rates were $60 \%$ TIMI III, 36\% TIMI II and 4\% TIMI I. The devices used to achieve revascularization in 48 patients were: Penumbra 054 $(n=3)$, Penumbra $041(n=16)$, Penumbra $032(n=14)$, Penumbra

Table 1 Baseline characteristics of study group

\begin{tabular}{ll}
\hline Characteristic & Overall \\
\hline $\mathrm{N}$ & 53 \\
Mean age (years) & 65.0 \\
Median age (years) & 66.0 \\
Gender, M/F (\%) & $49 / 51$ \\
Mean admission NIHSS & 17.2 \\
Median admission NIHSS & 17.0 \\
Location of vascular occlusion (\%) & \\
$\quad$ Internal carotid artery & 22.6 \\
$\quad$ Middle cerebral artery & 60.4 \\
$\quad$ Posterior circulation & 17.0 \\
Received intravenous tPA prior to & 34 \\
intervention (\%) & \\
\hline NIHSS, National Institute of Health Stroke Scale Score; tPA, tissue \\
plasminogen activator.
\end{tabular}


$026(n=4)$, balloon angioplasty $(n=7)$, stent $(n=3)$ and Merci L4 $(n=1)$.

Overall, eight patients (15\%) had bleeding complications including subarachnoid hemorrhage, parenchymal hemorrhage and intraventricular hemorrhage. Four of these patients had sICH giving an incidence of $8 \%$. There were three procedurerelated complications which included two (3.7\%) intracranial vessel perforations and a non- flow limiting carotid dissection related to guide catheter excursion. Follow-up NCCT scans at $24 \mathrm{~h}$ demonstrated SAH in an additional two patients, which were probably due to unrecognized procedural dissections or perforations. Hyperdensity in the basal ganglia was noted on five $24 \mathrm{~h}$ post-procedural CT scans. These hyperdensities all resolved the following day and were felt to represent contrast staining of the basal ganglia rather than hemorrhage.

Patients were initially selected for endovascular procedures based on CTP criteria and then analyzed based on time from symptom onset to procedure. The patients were divided into two groups: $\leq 6 \mathrm{~h}$ and $>6 \mathrm{~h}$ from symptom onset to neurointerventional procedure. There were no significant differences in age, race or gender between the groups (table 2). The mean NIHSS scores were 19.1 and $15.3(p=0.025)$, respectively, for the two groups. For the two time periods, $38.5 \%$ and $40.7 \%$ achieved a 90 -day $\mathrm{mRS} \leq 2(\mathrm{p}=1.0)$, and $57.7 \%$ and $51.9 \%(\mathrm{p}=0.785)$ achieved a 90 -day $m R S \leq 3$ (table 2). In addition, the percentage mortality as measured by an mRS of 6 at 90-day follow-up was 15.4 versus $29.6(\mathrm{p}=0.327)$ and the rate of ICH was 11.5 versus $18.5(p=0.704)$ for the two groups (table 2$)$.

When recanalizations were categorized into complete, partial or no recanalization, the $\leq 6 \mathrm{~h}$ group achieved recanalization rates of $61.5 \%, 38.5 \%$, and $0 \%$, respectively. In the $>6 \mathrm{~h}$ time group the percentages of complete, partial and no recanalization were $59.3 \%, 33.3 \%$, and $7.4 \%$, respectively ( $p=0.362$, table 2 ). Location of vessel occlusion was a consideration in the analysis for both groups. In the $\leq 6 \mathrm{~h}$ time group the rates of internal carotid artery, middle cerebral artery and posterior circulation (basilar artery and posterior inferior cerebellar artery) occlusions were $23.1 \%, 61.5 \%$, and $15.4 \%$, respectively compared with $22.2 \%, 59.3 \%$ and $18.5 \%$, respectively, in the $>6 \mathrm{~h}$ time group $(\mathrm{p}=0.955$, table 2$)$.

\section{DISCUSSION}

Our study demonstrated similar rates of good functional outcome and ICH in patients with ischemic stroke when endovascular treatment was performed based on CTP selection rather than time-guided selection. Our findings suggest that endovascular reperfusion in ischemic stroke may be effective and safe, and may allow patient selection not solely based on time from symptom onset. The decision to treat patients with acute ischemic stroke beyond the current recommended time intervals remains a challenge. Over the last several years our approach to stroke patients has considered physiologic imaging parameters rather than the strict conventional time criteria. We found that patients treated late (beyond $6 \mathrm{~h}$ ) had no difference in outcomes from those treated early (within $6 \mathrm{~h}$ ) if they were selected for treatment based on our physiologic imaging approach.

Intravenous tPA remains the mainstay of treatment for acute ischemic stroke. While it has been shown to be safe and relatively effective, many limitations exist. Only $4 \%$ of patients with acute stroke receive intravenous tPA, mostly related to patients presenting beyond the $3 \mathrm{~h}$ time window. ${ }^{20}$ Among patients presenting within the $3 \mathrm{~h}$ time window, almost half are ineligible to receive intravenous tPA owing to exclusion criteria. Furthermore, intravenous tPA does not perform well in recanalizing and sustaining recanalization in large vessel occlusions. ${ }^{21}$

Revascularization is associated with a 4.5-fold increase in the likelihood of a good clinical outcome. ${ }^{22}$ The PROACT II trial first documented that stroke patients with successful recanalization $(66 \%$ of the trial patients vs $18 \%$ of those with no recanalization, $\mathrm{p}=0.001)$ had better clinical outcomes, defined as $\mathrm{mRS} \leq 2(40 \%$ of those recanalized vs $25 \%$ of those achieving no recanalization, $p=0.04){ }^{6}$ This was further supported by subgroup analysis in the multi-Merci trial in which $49 \%$ of

Table 2 Comparison of treatment and outcomes between the two study groups: those treated $\leq 6 \mathrm{~h}$ versus those treated $>6 \mathrm{~h}$ from symptom onset

\begin{tabular}{|c|c|c|c|}
\hline \multirow[b]{2}{*}{ Endpoints } & \multicolumn{2}{|l|}{ Time to treatment } & \multirow[b]{2}{*}{ p Value } \\
\hline & $\leq 6 h(N=26)$ & $>6 \mathrm{~h}(\mathrm{~N}=27)$ & \\
\hline Mean age (years) & 65 & 65 & 0.968 \\
\hline \multicolumn{4}{|l|}{ Race } \\
\hline African American & $8(30.8)$ & $12(44.4)$ & 0.385 \\
\hline Caucasian American & $17(65.4)$ & $15(55.6)$ & \\
\hline Hispanic American & $1(3.8)$ & 0 & \\
\hline Gender, M/F (\%) & $12 / 14(46.2 / 53.8)$ & $14 / 13(51.9 / 48.1)$ & 0.786 \\
\hline Median time to treatment (h) & 4.0 & 8.5 & $<0.0001$ \\
\hline 90-day $m R S \leq 2$ & $10(38.5)$ & $11(40.7)$ & 1.0 \\
\hline 90-day $\mathrm{mRS} \leq 3$ & $15(57.7)$ & $14(51.9)$ & 0.785 \\
\hline Mortality & $4(15.4)$ & $8(29.6)$ & 0.327 \\
\hline Intravenous tPA & $13(50.0)$ & $5(18.5)$ & 0.021 \\
\hline \multicolumn{4}{|l|}{ Recanalization } \\
\hline Complete & $16(61.5)$ & $16(59.3)$ & 0.362 \\
\hline Partial & $10(38.5)$ & $9(33.3)$ & \\
\hline None & 0 & $2(7.4)$ & \\
\hline Mean NIHSS & 19.1 & 15.3 & 0.025 \\
\hline \multicolumn{4}{|l|}{ Location of occlusion } \\
\hline Internal carotid artery & $6(23.1)$ & $6(22.2)$ & 0.955 \\
\hline Middle cerebral artery & $16(61.5)$ & $16(59.3)$ & \\
\hline Posterior circulation & $4(15.4)$ & $5(18.5)$ & \\
\hline Intracranial hemorrhage & $3(11.5)$ & $5(18.5)$ & 0.704 \\
\hline
\end{tabular}

mRS, modified Rankin Score; NIHSS, National Institute of Health Stroke Scale Score; tPA, tissue plasminogen activator. 
revascularized patients achieved $m R S \leq 2$ compared with $10 \%$ of non-revascularized patients $(p<0.001)$. This study also showed a significant increase in 90-day mortality in patients who were not revascularized compared with those who were able to be revascularized $(52 \%$ vs $25 \%, \mathrm{p}<0.001) .{ }^{23}$ Similarly, the Penumbra POST Trial found that $45 \%$ of patients who were revascularized achieved a $\mathrm{mRS} \leq 2$ compared with $13 \%$ of patients who were not successfully recanalized. We demonstrated similar rates of good functional outcome regardless of whether patients were treated endovascularly before or after $6 \mathrm{~h}$ from symptom onset. Our study found that $38.5 \%$ and $40.7 \%$ of patients treated before and after $6 \mathrm{~h}$, respectively, achieved a 90-day $\mathrm{mRS} \leq 2$. Also, when recanalization is considered, all patients treated $\leq 6 \mathrm{~h}$ from symptom onset achieved either partial or total recanalization. For patients treated $>6 \mathrm{~h}$ from symptom onset, $93 \%$ achieved either partial (TIMI II) or total recanalization (TIMI III).

Revascularization is crucial for improved outcomes, so appropriate patient selection for revascularization therapies is paramount. Non-contrast CT scanning of the head has been the primary imaging modality in evaluating patients with acute stroke as it can screen for ICH and demonstrate early ischemic changes that might prevent patients from receiving intravenous tPA. ${ }^{24} 25$ The pattern of ischemic changes may be able to predict patients who will have a good functional outcome using the Alberta Stroke Program Early CT Score. ${ }^{26}$ However, the widespread acceptance of NCCT and the Alberta Stroke Program Early CT Score alone for stroke triage has been limited. This probably relates to the subtlety of acute ischemia in many instances on non-contrast CT scanning that can be difficult to detect.

The use of CTP has been advocated over the last decade as a physiologic tool and, when used in conjunction with noncontrast CT scanning, can identify the area of infarction through CBV maps and further define the penumbral region by integrating MTT data. ${ }^{27}$ The use of this physiologic approach to select patients within a specified time window is currently being studied in several randomized trials. ${ }^{28-30}$ This approach is also being applied to everyday practice to help select patients who present outside conventional time windows. ${ }^{31}$ We have also used this technique over the last several years to triage all stroke patients for potential intra-arterial therapy. We found that using CTP for selecting stroke patients allowed us to treat many patients who would not otherwise be considered candidates for therapy. This is best understood by comparing the two groups based on median time to presentation of $\leq 6 \mathrm{~h}$ or $>6 \mathrm{~h}$. When imaging criteria are used, $38.5 \%$ of patients treated $\leq 6 \mathrm{~h}$ from symptom onset achieved $\mathrm{mRS} \leq 2$ and $40.7 \%$ of patients treated $>6 \mathrm{~h}$ from symptom onset achieved $\mathrm{mRS} \leq 2(p=1.0)$. If we broaden our definition of good outcome to $\mathrm{mRS} \leq 3$, which still portends a functional lifestyle, in acute ischemic stroke the same trend continues with $57.7 \%$ and $51.9 \%$ of patients treated $\leq 6 \mathrm{~h}$ and $>6 \mathrm{~h}$, respectively, achieving 90 -day $\mathrm{mRS} \leq 3(\mathrm{p}=0.785) .{ }^{32} 33$

Our overall ICH rate was $15 \%$ with $8 \%$ sICH. This compares favorably with that seen in the previous device trials as well as reports from investigators treating similar patient populations. ${ }^{34}$ When stratifying our patient population to $\leq 6 \mathrm{~h}$ versus $>6 \mathrm{~h}$, we found similar rates of ICH $(11.5 \%$ vs $18.5 \%, p=0.704)$ and mortality ( $15.4 \%$ vs $29.6 \%, \mathrm{p}=0.327)$. While significance was not achieved, the overall small size of our study population and the even smaller number of patients experiencing ICH or death limit our ability to conclude whether or not patients treated $>6 \mathrm{~h}$ from symptom onset achieved statistically similar rates of $\mathrm{ICH}$ and death as those treated $\leq 6 \mathrm{~h}$. We recommend further study with a larger patient cohort to determine if rates of $\mathrm{ICH}$ and death are in fact similar between the two groups.

Our overall mortality rate was $23 \%$ which is similar to that in previous trials (HAMLET, DECIMAL, DESTINY). ${ }^{20} 3435$ Additionally, the median admission NIHSS score was 17, demonstrating that our patient selection was not heavily weighted towards those with lower severity stroke. The causes for the favorable result probably relate to patient selection, where we followed prior European Cooperative Acute Stroke Study (ECASS) recommendations and excluded patients with large infarctions (more than one-third MCA territory) and selected those with relatively large penumbral volume as evidenced by CTP. Performing thrombectomy primarily with a device and then the relatively judicious use of intra-arterial tPA also probably contributed to the low rates of $\mathrm{ICH}$. Close management in a dedicated neurocritical care unit may further contribute to improved outcomes.

Other limitations of this study include the retrospective nature of the data collection in a prospectively maintained patient database. Some patients had follow-up done by telephone interview and beyond 90 days, which could allow a bias to better outcome. The fact that we did not track the outcomes of the patients that we excluded based on penumbral imaging meant we were unable to compare the outcome with this group. Also, the significant differences in the mean admission NIHSS score between the $\leq 6 \mathrm{~h}$ and $>6 \mathrm{~h}$ patient groups (19.1 vs 15.3, $\mathrm{p}=0.025$ ) demonstrates that, while those in the later time group had statistically different NIHSS scores, this difference is less significant from a clinical standpoint since the mean scores in both groups were highly indicative of increased stroke severity. Furthermore, a previous study that stratified admission NIHSS score of $\leq 7$ versus $>7$ determined that those with an admission NIHSS $>7$ (as in our patient cohort) were less likely to improve neurologically and functionally. ${ }^{36}$

In addition, the use of perfusion imaging for patient triage could potentially bias the early group as some patients may have been excluded who otherwise would be included in comparative studies based on time parameters. The lack of a quantifiable methodology of CTP data for patient selection and the qualitative application of this approach by multiple physicians may weaken its applicability.

The practical application of physiologic imaging to select patients for acute stroke interventions irrespective of time was safe and effective in our single-center experience, with patient outcomes similar to those seen in other trials of acute stroke. The strongest evidence is the similar rates of good clinical outcomes ( $\mathrm{mRS} \leq 2$ and $\leq 3$ ) in patients treated $\leq 6 \mathrm{~h}$ compared with those treated $>6 \mathrm{~h}$ from symptom onset. The application of this approach may have a significant impact on the number of patients eligible for beneficial interventional therapy, especially those initially presenting to rural or community hospitals who are often ineligible for thrombolytic treatment.

\section{Competing interests None.}

Ethics approval This study was conducted with the approval of the Medical University of South Carolina Institutional Review Board (exempt status).

Provenance and peer review Not commissioned; externally peer reviewed.

\section{REFERENCES}

1. Anon. Tissue plasminogen activator for acute ischemic stroke. The National Institute of Neurological Disorders and stroke rt-PA stroke study group. N Engl J Med 1995;333:1581-7.

2. Hacke W, Kaste $M$, Fieschi $C$, et al. Intravenous thrombolysis with recombinant tissue plasminogen activator for acute hemispheric stroke. The European Cooperative Acute Stroke Study (ECASS). JAMA 1995;274:1017-25. 
3. Hacke W, Kaste M, Fieschi C et al. Randomised double-blind placebo-controlled trial of thrombolytic therapy with intravenous alteplase in acute ischaemic stroke (ECASS II). Second European-Australasian Acute Stroke Study Investigators. Lancet 1998:352:1245-51.

4. Hacke W, Kaste M, Bluhmki E, et al. Thrombolysis with alteplase 3 to 4.5 hours after acute ischemic stroke. N Engl J Med 2008;359:1317-29.

5. IMS II Trial Investigators. The Interventional Management of Stroke (IMS) II study. Stroke 2007;38:2127-35.

6. Furlan A, Higashida R, Wechsler L, et al. Intra-arterial prourokinase for acute ischemic stroke. The PROACT II study: a randomized controlled trial. Prolyse in Acute Cerebral Thromboembolism. JAMA 1999;282:2003-11.

7. Lin R, Vora N, Zaidi S, et al. Mechanical approaches combined with intra-arterial pharmacological therapy are associated with higher recanalization rates than either intervention alone in revascularization of acute carotid terminus occlusion. Stroke 2009:40:2092-7.

8. Shi ZS, Loh Y, Walker G, et al. Clinical outcomes in middle cerebral artery trunk occlusions versus secondary division occlusions after mechanical thrombectomy: Pooled analysis of the Mechanical Embolus Removal in Cerebral Ischemia (MERCI) and multi MERCI trials. Stroke 2010;41:953-60.

9. Smith WS, Sung G, Starkman S, et al. Safety and efficacy of mechanical embolectomy in acute ischemic stroke: results of the MERCI trial. Stroke 2005;36:1432-8.

10. Adams HP Jr, del Zoppo G, Alberts MJ, et al. Guidelines for the early management of adults with ischemic stroke: a guideline from the American Heart Association/ American Stroke Association Stroke Council, Clinical Cardiology Council,

Cardiovascular Radiology and Intervention Council, and the Atherosclerotic Peripheral Vascular Disease and Quality of Care Outcomes in Research Interdisciplinary Working Groups: the American Academy of Neurology affirms the value of this guideline as an educational tool for neurologists. Stroke 2007;38:1655-711.

11. Abou-Chebl A. Endovascular treatment of acute ischemic stroke may be safely performed with no time window limit in appropriately selected patients. Stroke 2010;41:1996-2000.

12. Schellinger PD, Thomalla G, Fiehler J, et al. MRI-based and CT-based thrombolytic therapy in acute stroke within and beyond established time windows: an analysis of 1210 patients. Stroke 2007;:38:2640-5.

13. Kohrmann $\mathbf{M}$, Juttler $\mathrm{E}$, Fiebach JB, et al. MRI versus CT-based thrombolysis treatment within and beyond the $3 \mathrm{~h}$ time window after stroke onset: a cohort study. Lancet Neurol 2006;5:661-7.

14. Koenig M, Kraus M, Theek C, et al. Quantitative assessment of the ischemic brain by means of perfusion-related parameters derived from perfusion CT. Stroke 2001;32:431-7

15. Wintermark $\mathbf{M}$, Flanders $A E$, Velthuis $B$, et al. Perfusion-CT assessment of infarct core and penumbra: receiver operating characteristic curve analysis in 130 patients suspected of acute hemispheric stroke. Stroke 2006;37:979-85.

16. Wintermark M, Rowley HA, Lev MH. Acute stroke triage to intravenous thrombolysis and other therapies with advanced ct or MR imaging: pro CT. Radiology 2009:251:619-26.

17. Ledezma CJ, Wintermark M. Multimodal CT in stroke imaging: new concepts. Radiol Clin North Am 2009;47:109-16.

18. Ezzeddine MA, Lev MH, McDonald CT, et al. CT angiography with whole brain perfused blood volume imaging: added clinical value in the assessment of acute stroke. Stroke 2002;33:959-66.
19. Lev MH, Segal AZ, Farkas J, et al. Utility of perfusion-weighted CT imaging in acute middle cerebral artery stroke treated with intra-arterial thrombolysis: prediction of final infarct volume and clinical outcome. Stroke 2001;32:2021-8.

20. Kleindorfer D, Kissela B, Schneider A, et al. Eligibility for recombinant tissue plasminogen activator in acute ischemic stroke: a population-based study. Stroke 2004:35:e27-9.

21. Christou I, Felberg RA, Demchuk AM, et al. Intravenous tissue plasminogen activator and flow improvement in acute ischemic stroke patients with internal carotid artery occlusion. J Neuroimaging 2002:12:119-23.

22. Rha JH, Saver JL. The impact of recanalization on ischemic stroke outcome: a metaanalysis. Stroke 2007;38:967-73.

23. Smith WS, Sung G, Saver J, et al. Mechanical thrombectomy for acute ischemic stroke: final results of the Multi MERCI trial. Stroke 2008;39:1205-12.

24. Schellinger PD, Fiebach JB, Hacke W. Imaging-based decision making in thrombolytic therapy for ischemic stroke: present status. Stroke 2003: 34:575-83.

25. Hacke W, Donnan G, Fieschi C et al. Association of outcome with early stroke treatment: pooled analysis of ATLANTIS, ECASS, and NINDS rt-PA stroke trials. Lancet 2004;363:768-74.

26. Barber PA, Demchuk AM, Zhang J, et al. Validity and reliability of a quantitative computed tomography score in predicting outcome of hyperacute stroke before thrombolytic therapy. Aspects study group. Alberta Stroke Programme Early CT Score. Lancet 2000;355:1670-4.

27. Rowley HA, Roberts TP. Clinical perspectives in perfusion: neuroradiologic applications. Top Magn Reson Imaging 2004;15:28-40.

28. Hacke W, Albers G, Al-Rawi Y, et al. The Desmoteplase In Acute ischemic Stroke trial (DIAS): a phase II MRI-based 9-hour window acute stroke thrombolysis trial with intravenous desmoteplase. Stroke 2005;36:66-73.

29. Lansberg MG, Thijs VN, Hamilton S, et al. Evaluation of the clinical-diffusion and perfusion-diffusion mismatch models in defuse. Stroke 2007;38:1826-30.

30. De Silva DA, Brekenfeld C, Ebinger $M$, et al. The benefits of intravenous thrombolysis relate to the site of baseline arterial occlusion in the Echoplanar Imaging Thrombolytic Evaluation Trial (EPITHET). Stroke 2010;41:295-9.

31. Natarajan SK, Snyder KV, Siddiqui AH, et al. Safety and effectiveness of endovascular therapy after 8 hours of acute ischemic stroke onset and wake-up strokes. Stroke 2009; 40:3269-74.

32. Juttler E, Schwab S, Schmiedek P, et al. Decompressive surgery for the Treatment of Malignant Infarction of the Middle Cerebral Artery (DESTINY): a randomized, controlled trial. Stroke 2007:38:2518-25.

33. Hofmeijer J, Kappelle LJ, Algra A, et al. Surgical decompression for spaceoccupying cerebral infarction (the hemicraniectomy after middle cerebral artery infarction with Life-Threatening Edema Trial [HAMLET]): a multicentre, open, randomised trial. Lancet Neurol 2009;8:326-33.

34. Shi ZS, Loh Y, Walker G, et al. Endovascular thrombectomy for acute ischemic stroke in failed intravenous tissue plasminogen activator versus non-intravenous tissue plasminogen activator patients: revascularization and outcomes stratified by the site of arterial occlusion. Stroke 2010:41:1185-92.

35. The Penumbra Pivotol Stroke Trial Investigators. The Penumbra pivotol stroke trial. Stroke 2009;40:2761-8.

36. DeGraba T, Hallenbeck J, Pettigrew $\mathrm{K}$, et al. Progression in acute stroke: value of the initial NIH stroke scale score on patient stratification in future trials. Stroke 1999;30:1208-12. 